VOL. 20 (1979), 139-143.

\title{
Continuity of Cima and Rung's extension and non normal meromorphic functions
}

\section{Douglas M. Campbell}

A function meromorphic in $|z|<1$ is constructed such that on every curve in $|z|<1$ which goes to $|z|=1$ the set of limit points of the function is the entire complex plane. This example is used to prove the existence of non-normal meromorphic functions in $|z|<1$ which have continuous set valued extensions. Cima and Rung had introduced a set valued extension for meromorphic functions and proved that all normal meromorphic functions have a continuous extension while all functions with a continuous extension have the Lindelöf property. For a long time it was thought that this might characterize normal meromorphic functions. This paper proves that it is not possible to determine the normality of a meromorphic function by the continuity of Cima and Rung's set valued extension. The paper closes with the open problem: do there exist non-normal conalytic functions for which Cima and Rung's set valued extension is continuous?

Cima and Rung in 1966 investigated the relation between normal functions and a class of associated boundary functions [4]. Let $\Lambda$ be the family of all continuous curves $\lambda(t)$ with values in $|z|<1$, and $\lim |\lambda(t)|=1$. A meromorphic function $f$ induces a mapping $\hat{f}$ on $\Lambda$ $t \rightarrow 1$

by setting $\hat{f}(\lambda)=c(f, \lambda)$ where $C(f, \lambda)$, the cluster set of $f$ on $\lambda$, is the set of all $w$ in $\mathbb{G} \cup\{\infty\}=\hat{\mathbb{U}}$ such that there is a sequence $t_{n} \rightarrow 1$ with $f\left(\lambda\left(t_{n}\right)\right) \rightarrow w$. They proved that this extension is continuous Received 2 January 1979. 
if $f$ is normal and if this extension is continuous then $f$ must have the Lindelöf property, a property possessed by all normal meromorphic functions.

Five years later Brown and Gauthier [3] proved that $f$ is normal if and only if $f$ can be continuously extended to the regular points of the maximal ideal space of $H^{\infty}$. It is therefore reasonable to ask whether Cima and Rung's machinery provides a similar characterization; that is, is $f$ normal if and only if Cima and Rung's extension is continuous?

The purpose of this paper is to show that the continuity of their extension does not characterize normal meromorphic functions by proving the existence of a very bad meromorphic function with $\hat{f}(\lambda) \equiv \hat{\mathbb{l}}$. We first give an application of the so called pole pushing technique. The following version of Mergelyan's Theorem is not as well known as the polynomial version $[6,427, \# 1]$.

MERGELYAN'S THEOREM. Let $K$ be a compact set in $\mathbb{\mathfrak { l }}$ with $\mathbb{\mathbb { a }}-K$ of finite connectivity. If $f(z)$ is continuous on $K$ and onalytic on the interior of $K$, then for every $\varepsilon>0$, there is a rational function $R(z)$ such that $|f(z)-R(z)|<\varepsilon$ on $K$.

POLE PUSHING LEMMA. Let $\left\{r_{n}\right\}_{n=1}^{\infty}$ be any sequence of positive strictly increasing real numbers tending to infinity and $\left\{b_{n}\right\}_{n=1}^{\infty}$ be any sequence of points of $\mathbb{a}$. Then there exists a meromorphic function $R(z)$ in a such that $\lim _{n \rightarrow \infty} \max _{|z|=x_{n}}\left|R(z)-b_{n}\right|=0$.

Proof. We proceed to define $\left\{f_{n}(z)\right\}_{n=1}^{\infty},\left\{R_{n}(z)\right\}_{n=1}^{\infty}$ inductively. Let $f_{1}(z) \equiv R_{1}(z) \equiv b_{1}$ on $|z| \leq r_{1}$. Let $f_{2}(z)$ be $f_{1}(z)$ on $|z| \leq r_{1}$ and $b_{2}$ on $|z|=r_{2}$. By Mergelyan's Theorem there is a rational function $R_{2}(z)$ such that $\left|f_{2}(z)-R_{2}(z)\right| \leq 2^{-2}$ on $\left\{\left|z_{2}\right| \leq r_{1} \cup|z|=r_{2}\right\}$. Let $\{p(2, k)\}_{k=1}^{N_{2}}$ be the poles of $R_{2}(z)$ in $|z| \leq r_{2}$ and $P(2, k, z)$ be the principal part of $R_{2}(z)$ at $p(2, k)$. Let $f_{3}(z)$ be $R_{2}(z)$ on $|z| \leq r_{2}$ and $b_{3}$ on $|z|=r_{3}$. The function 
$g_{3}(z)=f_{3}(z)-\sum_{k=1}^{N_{2}} P(2, k, z)$ (with poles pushed out of the region of interest) satisfies Mergelyan's Theorem on $\left\{|z| \leq r_{2} \cup|z|=r_{3}\right\}$, and therefore there exists a rational function $S_{3}(z)$ such that $\left|g_{3}(z)-S_{3}(z)\right|<2^{-3}$ on $\left\{|z| \leq r_{2} \cup|z|=r_{3}\right\}$. Setting $R_{3}(z)=S_{3}(z)+\sum_{k=1}^{N_{2}} P(2, k, z)$ yields $\left|f_{3}(z)-R_{3}(z)\right| \leq 2^{-3}$ on $\left\{|z| \leq r_{2} \cup|z|=r_{3}\right\}$. In general let $f_{n}(z)$ be $R_{n-1}(z)$ on $|z| \leq r_{n-1}$ and $b_{n}$ on $|z|=r_{n}$. The function $g_{n}(z)=f_{n}(z)-\sum_{k=1}^{N_{n}} P(n-1, k, z)$ satisfies Mergelyan's Theorem on $\left\{|z| \leq r_{n-1} \cup|z|=r_{n}\right\}$. Therefore there exists a rational function $S_{n}(z)$ such that $\left|g_{n}(z)-S_{n}(z)\right|<2^{-n}$ on $\left\{|z| \leq r_{n-1} \cup|z|=r_{n}\right\}$. Letting $R_{n}(z)=S_{n}(z)+\sum_{k=1}^{N_{n}} P(n-1, k, z)$ shows $\left|f_{n}(z)-R_{n}(z)\right|<2^{-n}$ on $\left\{|z| \leq r_{n-1} \cup|z|=r_{n}\right\}$.

To show $R_{n}(z)$ converges uniformly on compacta of $\mathbb{a}$ it clearly suffices to show $\left\{R_{n}(z)\right\}$ is Cauchy on each set of the form $|z| \leq r_{m}$. This is immediate upon noting for all $k>m$ that, on $|z| \leq r_{m}$,

$$
2^{-k}>\left|f_{k}(z)-R_{k}(z)\right|=\left|R_{k-1}(z)-R_{k}(z)\right| .
$$

We now prove the main claim of the lemma. We note that

$$
\left|R(z)-b_{n}\right| \leq\left|R(z)-R_{m}(z)\right|+\left|R_{m}(z)-R_{n}(z)\right|+\left|R_{n}(z)-b_{n}\right| \text {. }
$$

By choosing $m$ large enough the first term on the right is less than $2^{-n}$ on $|z|=r_{n}$. The second term is less than

$$
\sum_{j=n}^{m-1}\left|R_{j+1}(z)-R_{j}(z)\right|<\sum_{j=n}^{\infty} 2^{-(j+1)}=2^{-n}
$$


By construction the third term is less than $2^{-n}$. Thus $\max _{|z|=r_{n}}\left|R(z)-b_{n}\right| \rightarrow 0$ as $n \rightarrow \infty$ as claimed.

THEOREM. There exists a non-normal meromorphic function $f(z)$ in $|z|<1$ such that $C(f, \gamma)=\hat{\mathbb{Q}}$ for every curve $\gamma(t), \lim _{t \rightarrow 1}|\gamma(t)|=1$.

Proof. Let $g(z)$ be any strongly annular function, that is $g(z)$ is analytic in $|z|<1$, and there is a sequence of increasing positive numbers $S_{n}, S_{n}<1$, such that $\lim _{n \rightarrow \infty}\left\{\min _{|z|=S_{n}}|g(z)|\right\}=\infty ;$ for example, $g(z)=\sum_{j=1}^{\infty}\left\{1-z /\left(1-j !^{-1}\right)\right\}^{j !} \quad$ (Bonar's monograph [2] is a rich source of examples). All annular functions are non-normal since they have Köbe arcs. Let $\left\{b_{n}\right\}$ be any sequence of complex numbers such that every point of $\hat{b}$ is a limit point of $\left\{b_{n}\right\}$ and let $R(z)$ be the associated meromorphic function of the Pole Pushing Lemma. Then the function $f(z)=R(g(z))$ is meromorphic in $|z|<1$. Any curve $\gamma(t)$ with $\lim |\gamma(t)|=1$ is mapped $t \rightarrow 1$

by $g(z)$ onto a curve with lim sup $g(\gamma(t))=\infty$. Since the lim sup of $g(\gamma(t))$ is infinite the curve $g(\gamma(t))$ crosses all but a finite number of the $r_{n}$ 's and therefore its cluster set must be $\hat{0}$. It is trivial to check that any meromorphic function of a non-normal function is non-normal. This concludes the the proof.

There is no possibility of finding a non-normal analytic function with this property since every unbounded analytic function has a curve $\gamma(t)$, $|\gamma(t)| \rightarrow 1$, on which $f$ has an asymptotic value.

Since $C(f, \gamma)$ is identically $\hat{\mathbb{d}}$ for all curves $\gamma$, the extension $\hat{f}$ is constant and automatically continuous. Thus there are non-normal meromorphic functions for which $\hat{f}$ is continuous which proves that the continuity of Cima and Rung's extension does not characterize normal meromorphic functions.

We close with an open problem: are there non-normal analytic functions for which $\hat{f}$ is continuous? 


\section{References}

[1] K.F. Barth, "Asymptotic values of meromorphic functions", Michigan Math. J. 13 (1966), 321-340.

[2] D.D. Bonar, on annular functions (Mathematische Forschungsberichte, 24. VEB Deutscher Verlag der Wissenschaften, Berlin, 1971).

[3] Leon Brown and Paul Gauthier, "Behavior of normal meromorphic functions on the maximal ideal space of $\mathrm{H}^{\infty} "$, Michigan Math. J. 18 (1971), 365-371.

[4] J.A. Cima and D.C. Rung, "Normal functions and a class of associated boundary functions", Israel J. Math. 4 (1966), 119-126.

[5] OIIi Lehto and K.l. Virtanen, "Boundary behaviour and normal meromorphic functions", Acta Math. 97 (1957), 47-65.

[6] Walter Rudin, Real and complex analysis, Ind edition (MacGraw-Hill, New York, St. Louis, San Francisco, Toronto, London, Sydney, 1974).

Department of Mathematics, University of Michigan,

Ann Arbor,

Michigan,

USA. 